\title{
Norovirus antagonism of B-cell antigen presentation results in impaired control of acute infection
}

\author{
S Zhu ${ }^{1}$, MK Jones ${ }^{1}$, D Hickman ${ }^{1}, S$ Han $^{2}$, W Reeves ${ }^{2}$ and SM Karst ${ }^{1}$
}

Human noroviruses are a leading cause of gastroenteritis, and so, vaccine development is desperately needed. Elucidating viral mechanisms of immune antagonism can provide key insight into designing effective immunization platforms. We recently revealed that $B$ cells are targets of norovirus infection. Because noroviruses can regulate antigen presentation by infected macrophages and $\mathrm{B}$ cells can function as antigen-presenting cells, we tested whether noroviruses regulate B-cell-mediated antigen presentation and the biological consequence of such regulation. Indeed, murine noroviruses could prevent B-cell expression of antigen presentation molecules and this directly correlated with impaired control of acute infection. In addition to B cells, acute control required MHC class I molecules, CD8 ${ }^{+} \mathrm{T}$ cells, and granzymes, supporting a model whereby B cells act as antigen presenting cells to activate cytotoxic CD8 ${ }^{+} \mathrm{Tcells}$ This immune pathway was active prior to the induction of antiviral antibody responses. As in macrophages, the minor structural protein VP2 regulated B-cell antigen presentation in a virus-specific manner. Commensal bacteria were not required for the activation of this pathway and ultimately only $B$ cells were required for the clearance of viral infection. These findings provide new insight into the role of $\mathrm{B}$ cells in stimulating antiviral $\mathrm{CD}^{+}{ }^{+} \mathrm{T}$-cell responses.

\section{INTRODUCTION}

Human noroviruses (HuNoVs) are a significant cause of gastroenteritis outbreaks across the globe. Since the introduction of effective rotavirus vaccines, they have become the leading cause of severe childhood diarrhea in the United States, ${ }^{1,2}$ a trend that is likely true globally. ${ }^{3,4}$ They are also the principal cause of foodborne disease outbreaks. ${ }^{5}$ Recent data suggest that $\mathrm{HuNoV}$ infections in children under 5 years of age cause approximately one million annual health-care visits and cost over $\$ 273$ million in the United States alone, ${ }^{2}$ and that foodborne $\mathrm{HuNoV}$ infections cost $\$ 6$ billion each year. ${ }^{6}$ Overall, the disease burden caused by these enteric pathogens is extremely high and the development of $\mathrm{HuNoV}$ vaccines is a critical need. A major concern in $\mathrm{HuNoV}$ vaccine development is that natural infection fails to elicit long-lasting protective immunity. ${ }^{7-9}$ The basis of this suboptimal memory immune response is unclear, and so, it is difficult to predict whether $\mathrm{HuNoV}$ vaccines will suffer from the same immunological insufficiency. Initial results of clinical trials testing $\mathrm{HuNoV}$ virus-like particles as vaccines indicate that they provide modest protection from severe disease during a live virus challenge if the infection occurs within 1 month of immunization. ${ }^{10,11}$ However, virus-specific antibody responses elicited by the virus-like particles waned within 6 months. ${ }^{12}$ Elucidating NoV interactions with the host immune system, and particularly their mechanisms of immune evasion and antagonism, should inform the development of next-generation vaccine candidates. ${ }^{13}$

Several HuNoV proteins block host secretory pathways which could prevent cytokine secretion from infected cells. ${ }^{14,15}$ However, the relevance of putative immune antagonism strategies cannot be easily addressed for HuNoVs because of the lack of an immunocompetent and genetically tractable animal model system: HuNoVs infect severely immunodeficient mice, ${ }^{16}$ but this is not a tenable system for dissecting host immune responses. They also infect gnotobiotic piglets and

${ }^{1}$ College of Medicine, Department of Molecular Genetics \& Microbiology, Emerging Pathogens Institute, University of Florida, Gainesville, Florida, USA and ${ }^{2}$ College of Medicine, Department of Medicine, Division of Rheumatology \& Clinical Immunology, University of Florida, Gainesville, Florida, USA. Correspondence: SM Karst (skarst@ufl.edu) 
calves, ${ }^{17,18}$ but the germ-free nature of these models reduces their utility for immunology studies. Finally, chimpanzees can be asymptomatically infected, ${ }^{19}$ but this research is now restricted. In light of these limitations in studying $\mathrm{HuNoV}$ infection in animal models, others and we take advantage of the availability of murine NoVs (MuNoVs). ${ }^{20}$ The strengths and weaknesses of this model system have been recently reviewed in detail. ${ }^{21}$ Particularly relevant to our study are the similarities between immune responses to HuNoVs and MuNoVs: they are all modestly inflammatory ${ }^{18,22-27}$ and certain strains fail to elicit robust protective immunity. ${ }^{7-9,28,29}$ Several immune antagonism strategies have been identified using the MuNoV model system and their functions confirmed to influence in vivo infections: First, the MuNoV virulence factor 1 protein blocks cytokine expression and prevents apoptosis of infected macrophages; this activity regulates MNV-1 virulence. ${ }^{30}$ Second, the MuNoV minor structural protein VP2 prevents the upregulation of antigen presentation molecules in infected macrophages; this activity regulates protective immunity induction. $^{28}$

MuNoVs are well-established to infect macrophages and dendritic cells, ${ }^{31}$ and there is evidence that this is also true for HuNoVs although this has not been replicated in vitro. ${ }^{16,19,32,33}$ We recently demonstrated that HuNoVs and MuNoVs also infect B cells. ${ }^{34,35}$ Considering the ability of the MuNoV VP2 protein to regulate antigen presentation in macrophages in a virus strain-specific manner ${ }^{28}$ we were interested to determine whether VP2 similarly regulates antigen presentation by B cells. Indeed, we have revealed that the MuNoV VP2 protein can block the upregulation of antigen presentation molecules in B cells. Surprisingly though, this antagonist strategy did not correlate with protective immunity induction but instead prevented the activation of cytotoxic $\mathrm{CD} 8{ }^{+} \mathrm{T}$ cells that were critical in controlling acute MuNoV infection. Thus, VP2 regulation of antigen presentation occurs in a cell type- and virus strain-specific manner: the MNV-1 VP2 protein blocks antigen presentation in macrophages and this suppresses protective immunity induction; ${ }^{28}$ whereas the MNV-3 VP2 protein blocks antigen presentation in B cells and this prevents acute control (shown herein). The cell type specificity of VP2 immune antagonist activity thus dictates the overall immune outcome to a NoV infection and could be modulated to enhance the immunogenicity of next-generation vaccines.

\section{RESULTS \\ MNV-1, but not MNV-3, stimulates B cells to upregulate antigen presentation molecules}

Our previous results revealed that a $\mathrm{MuNoV}$ strain called MNV-3 stimulates antigen presentation by infected macrophages, resulting in the induction of robust protective immunity mediated by antiviral antibody and $\mathrm{CD} 44^{+} \mathrm{T}$ cells; whereas another strain called MNV-1 blocks antigen presentation in macrophages and thus fails to elicit robust protective immunity. ${ }^{28}$ We recently identified $\mathrm{B}$ cells as an additional target of NoV infection. ${ }^{34,35}$ Thus, we predicted a similar virus strain-specific phenotype would be observed in infected B cells, which can also serve as antigen-presenting cells (APCs). To test this, we measured the levels of antigen presentation molecules on infected $\mathrm{B}$ cells in vitro taking advantage of the fact that the M12 B-cell line is comparably permissive to MNV-1 and MNV-3 infections. ${ }^{34}$ To our surprise, MNV-1 infection stimulated M12 cells to upregulate MHC class I as well as the co-stimulatory molecules CD40, CD80, and CD86 on their surface (Figure 1a, gray bars). Surface expression of MHC class II molecules was not significantly upregulated by MNV-1 infection, although there was a similar trend compared with the other markers. None of the markers were upregulated when UV-inactivated MNV-1 was inoculated onto M12 cells (data not shown), demonstrating that viral replication was required for M12 cell maturation. In contrast to results with MNV-1, MNV-3 failed to stimulate surface expression of any antigen presentation molecule above basal levels (Figure 1a, white bars). These results are in direct opposition to our previous results in RAW 264.7 macrophages. ${ }^{28}$ Because of the surprising nature of these findings, we performed parallel M12 and RAW 264.7 cell infections with MNV-1 and MNV-3 and confirmed that MNV1 induced upregulation of antigen presentation molecules on $\mathrm{B}$ cells, whereas MNV-3 induced their upregulation on macrophages (Figure 1b, relative MHC class I expression on infected cells compared with mock-inoculated cells shown as a representative marker). We next tested whether MNV-1 and MNV-3 caused B cells to upregulate antigen presentation molecules during an in vivo infection. The same trend noted during M12 infections was observed in B6 mice: MNV-1 infection stimulated the surface expression of MHC class I and CD86 on Peyer's patch $\mathrm{CD} 19^{+} \mathrm{B}$ cells at 2 days post infection (d.p.i.) (Figure 1c, gray bars); although MNV-3 infection also upregulated MHC class I and CD86 on B cells, the levels were significantly lower than those induced by MNV-1 (Figure 1c, white bars). Neither virus strain caused an upregulation of MHC class II, CD40, or CD80, which may be because of the fact that upregulation of these molecules on $\mathrm{B}$ cells in vivo generally requires $\mathrm{CD} 40$ engagement with its cognate receptor on activated $\mathrm{CD}_{4}{ }^{+} \mathrm{T}$ cells. ${ }^{36}$ These data suggest that Peyer's patch $B$ cells upregulate certain antigen presentation molecules early after MuNoV infection in the absence of $\mathrm{CD} 4^{+} \mathrm{T}$-cell help, and the magnitude of upregulation is virus strain-specific.

Considering that MNV-1 does not induce robust protective immunity, ${ }^{28,29}$ we hypothesized that the stimulation of B-cellmediated antigen presentation has a critical role in the acute control of infection in contrast to induction of memory immunity. To first determine whether overall MHC class I- or MHC class II-mediated antigen presentation contributes to acute control of MuNoV infection, we measured virus titers at 3 d.p.i. in mice lacking $\beta 2$ microglobulin (B2M) or MHC class II (MHC II) molecules (Figure 1d): MNV-1 titers were significantly higher in the distal ileum and mesenteric lymph nodes (MLNs), but not the colon, of $\mathrm{B}_{2} \mathrm{M}^{-1-}$ mice compared with wild-type B6 mice, demonstrating that MHC class I antigen presentation is required for early MNV-1 control in a tissue-specific manner. MNV-3 titers in $\mathrm{B} 6$ and $\mathrm{B} 2 \mathrm{M}^{-/-}$mice 
a

\section{In vitro B-cell maturation as APC}

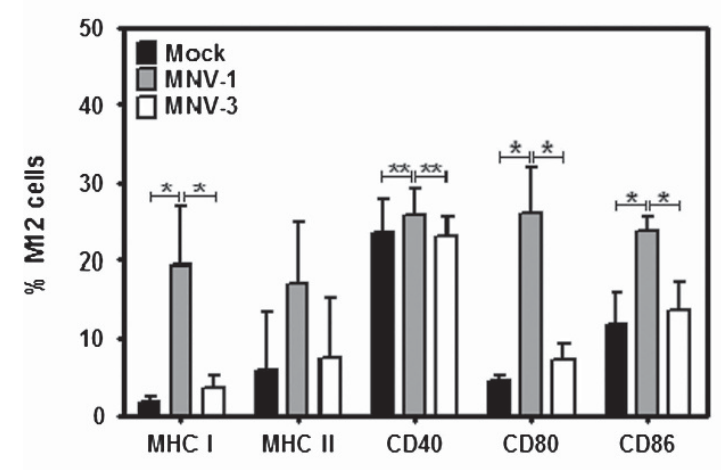

C

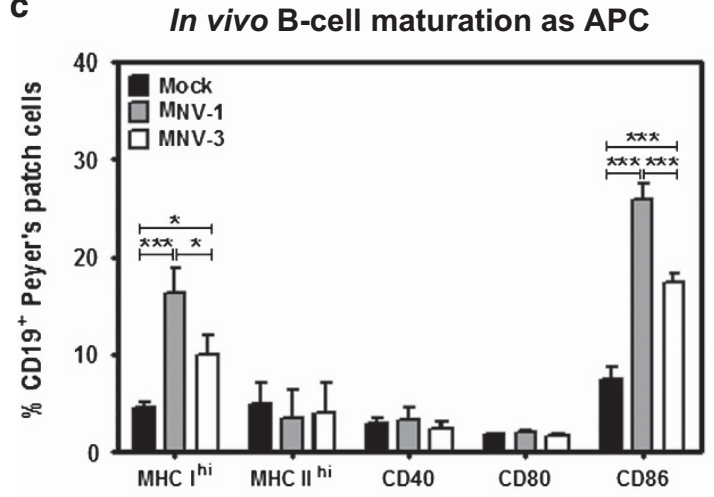

b

M12 vs. RAW 264.7 cells

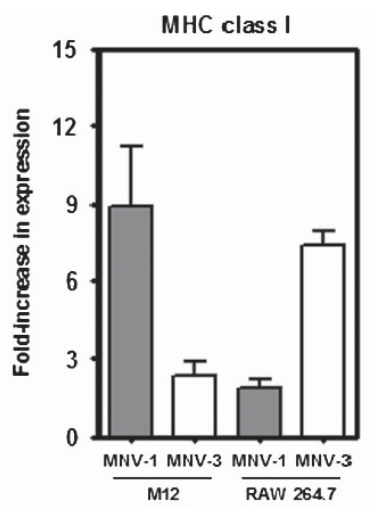

d

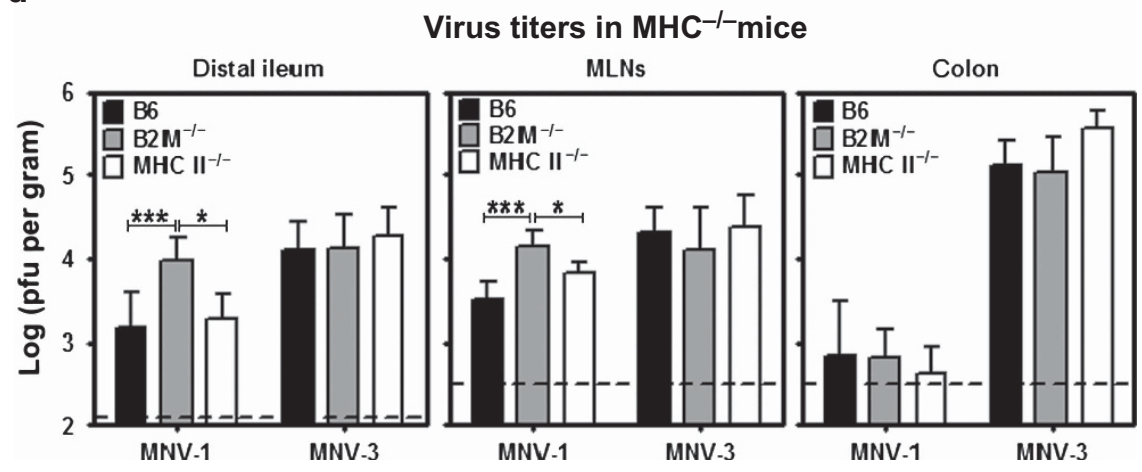

Figure 1 MNV-1, but not MNV-3, induces B cells to upregulate antigen presentation molecules. (a) Duplicate wells of M12 cells were inoculated with mock inoculum (black bars), MNV-1 (gray bars), or MNV-3 (white bars) at MOI 5. At 2 d.p.i., cell were stained with antibodies to MHC I, MHC II, CD40, $\mathrm{CD} 80$, and CD86. Flow cytometry was carried out as described in the Methods and isotype control antibodies were used to set gates. The experiment was repeated three times and data from all experimental replicates were averaged. Statistical comparisons were made between mock and each virus strain; and MNV-1 and MNV-3. (b) The same experiment described for panel A was repeated two additional times, performing parallel M12 and RAW 264.7 cell infections. RAW 264.7 cells were stained for surface markers at 1 d.p.i. because of the faster viral replication kinetics in this cell line. Data for MHC I levels are presented as the fold-difference in expression on infected cells compared to mock-inoculated cells. The same trend (increased expression on MNV-1infected M12 cells and MNV-3-infected RAW 264.7 cells) was observed for MHC II, CD40, CD80, and CD86 as well (data not shown). (c) Groups ( $n=2$ ) of B6 mice were mock-inoculated (black bars) or infected p.o. with $10^{7} \mathrm{TCID}_{50}$ units MNV-1 (gray bars) or MNV-3 (white bars). At 2 d.p.i., Peyer's patches were dissected, pooled for each mouse, and single cell suspensions were generated. Cells were stained for CD19, MHC I, MHC II, CD40, CD80, and CD86 and assessed by flow cytometry. Data are reported as the percentage of CD19 ${ }^{+}$cells expressing each antigen presentation molecule. The experiment was repeated three times and data from all experimental replicates were averaged. Statistical comparisons were made between mock and each virus strain; and MNV-1 and MNV-3. (d) Groups of B6 (black bars), B2M ${ }^{-1-}$ (gray bars), and MHC II ${ }^{-1-}$ (white bars) mice were infected p.0. with $10^{7} \mathrm{TCID}_{50}$ units MNV-1 or MNV-3, as indicated on the $x$ axis. At 3 d.p.i., the indicated tissues were dissected and viral titers were determined by plaque assay. The data are reported as pfu per gram of tissue and the data for all mice per group ( $n=6-8$ mice over three experiments) are averaged. Dashed lines indicate the limit of detection for each tissue. B6 mice were compared with $\mathrm{B} 2 \mathrm{M}^{-1-}$ and $\mathrm{MHC} \mathrm{II}{ }^{-I-}$ strains, and $\mathrm{B} 2 \mathrm{M}^{-/-}$and $\mathrm{MHC} \mathrm{II}^{-/-}$were compared with each other, for statistical purposes for each virus strain. d.p.i., days post infection; MOI, multiplicity of infection; p.o., perorally. 
were comparable in all three tissues. Moreover, MHC II was dispensable for MNV-1 control. Collectively, these data are consistent with a role for virally induced MHC class I-mediated antigen presentation by $\mathrm{B}$ cells in controlling acute $\mathrm{MuNoV}$ infection. They also suggest that MNV-3 prevents this process, resulting in impaired early control of infection.

\section{B cells control acute MuNoV infection in a virus strain-specific manner}

The data above demonstrate a correlation between the upregulation of antigen presentation molecules specifically on B cells and MHC class I-dependent acute control of MuNoV infection. We next directly examined the role of $\mathrm{B}$ cells in controlling acute $\mathrm{MuNoV}$ infections by infecting B6 or B-celldeficient $\mu \mathrm{MT}$ mice with MNV-1 or MNV-3 and measuring virus titers during the time frame in which acute infection is controlled. MNV-1 titers were significantly higher in $\mu \mathrm{MT}$ mice than in B6 mice at all time points 3-14 d.p.i. (Figure 2a). This phenotype was particularly notable at 3-5 d.p.i., where titers were markedly higher in the distal ileums (40-fold at 3 d.p.i. and 21 -fold at 5 d.p.i.), MLNs (11-fold and 23-fold), and colons (10fold and 6-fold) of $\mu \mathrm{MT}$ mice compared with their wild-type counterparts. B cells were also critical for MNV-1 clearance as indicated by residual virus titers at $7-14$ d.p.i. in $\mu \mathrm{MT}$ mice, but the phenotype was not as pronounced as at 3-5 d.p.i. In striking contrast, B cells played only a minor role in the control of acute MNV-3 infection: distal ileum and colon titers were comparable in $\mu \mathrm{MT}$ and B6 mice at 3-5 d.p.i., and there was only a fourfold increase in MLN titers of $\mu \mathrm{MT}$ mice at 3 d.p.i. (Figure 2b). B cells did contribute modestly to MNV-3 control at 10-14 d.p.i. but were not sufficient for clearance; this was expected because MNV-3 is capable of establishing a persistent infection in wild-type mice. ${ }^{37,38}$ Collectively, these data indicate a critical role for $\mathrm{B}$ cells in controlling $\mathrm{MNV}-1$ infection at 3-5 d.p.i., a time point preceding an expected antiviral antibody response. Importantly, this early role played by B cells was active against MNV-1, but not MNV-3, infection and thus correlates with the upregulation of antigen presentation molecules on B cells.

\section{Antiviral antibody production does not account for the function of B cells in controlling acute MNV-1 infection} Considering that $\mathrm{B}$ cells had their most notable effect in controlling MNV-1 infection at 3-5 d.p.i. and that we previously reported that $\mathrm{MNV}-3$ induces a much more robust antiviral antibody response than $\mathrm{MNV}-1,{ }^{28}$ it seemed unlikely that antiviral antibody production accounted for the dramatically increased virus titers in MNV-1-infected $\mu \mathrm{MT}$

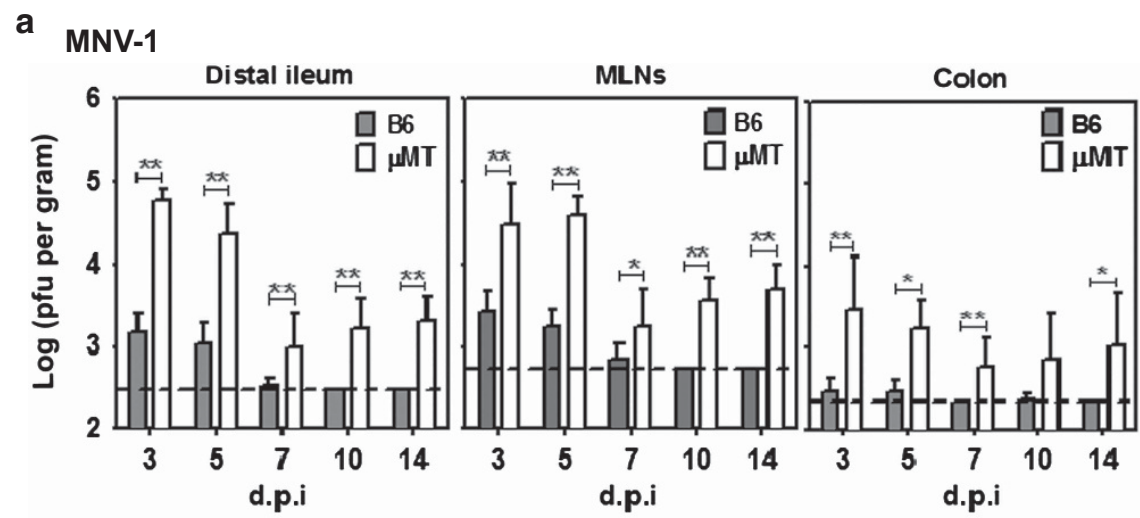

b MNV-3
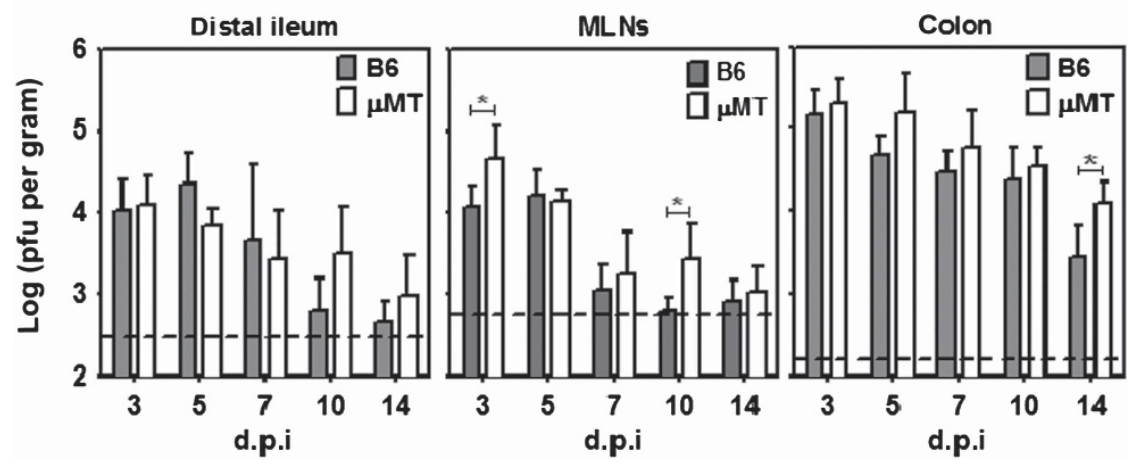

Figure 2 B cells control acute MNV-1, but not MNV-3, infection. Groups of B6 (gray bars) and $\mu$ MT (white bars) mice were infected p.o. with $10^{7}$ TCID 50 units MNV-1 (a) or MNV-3 (b). At 3, 5, 7, 10, and 14 d.p.i., the indicated tissues were dissected and virus titers were determined by plaque assay. The data are reported as pfu per gram of tissue and the data for all mice per condition ( $n=5-9$ mice over three experiments) are averaged. Dashed lines indicate the limit of detection for each tissue. The two mouse strains were compared at each time point for each tissue for statistical purposes. d.p.i., days post infection; p.o., perorally. 
a

$10^{4} \mathrm{TCID}_{50}$ units MuNoV
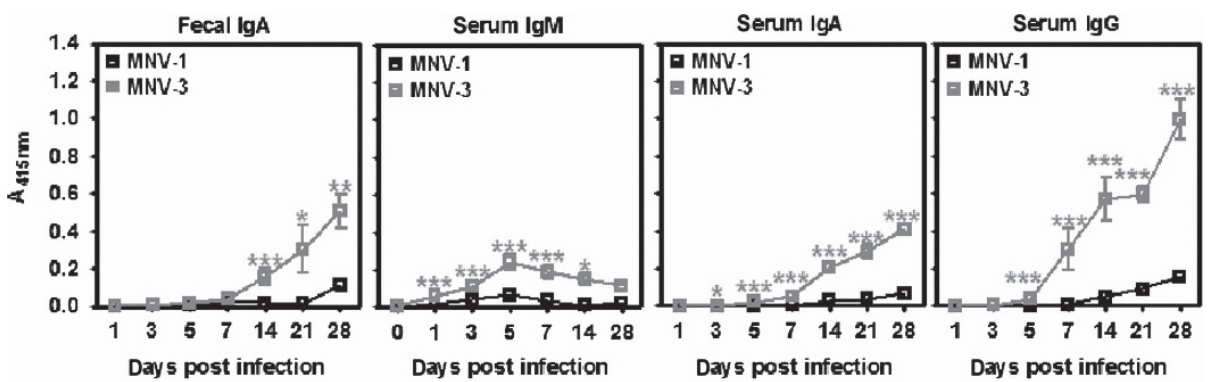

b

$10^{7} \mathrm{TCID}_{50}$ units MuNoV
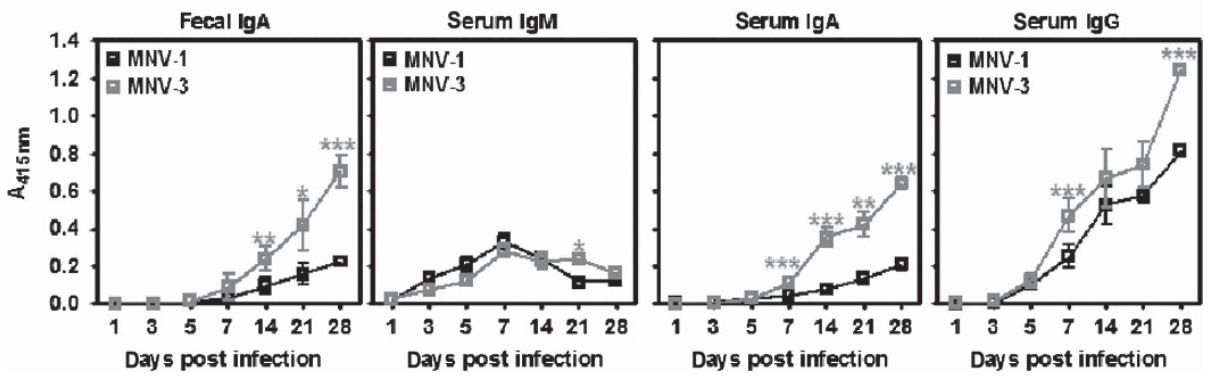

Figure 3 Antiviral antibody responses do not account for B-cell-mediated control of MNV-1 infection. Groups of B6 mice were infected p.o. with either $10^{4} \mathrm{TCID}_{50}$ units (a) or $10^{7} \mathrm{TCID}_{50}$ units (b) of MNV-1 (black lines) or MNV-3 (gray lines). At 0, 1, 3, 5, 7, 14, 21, and 28 d.p.i., fecal pellets and serum were collected from each mouse. Virus-specific antibody was detected by standard ELISA using an anti-mouse IgA secondary antibody for fecal lysates, and anti-mouse IgM, IgG, and IgA secondary antibodies for serum samples. The data are reported as the averaged absorbance readings for all mice per condition ( $n=6$ mice over two experiments) subtracted by the absorbance of samples collected at 0 d.p.i. The two virus strains were compared at each time point for statistical purposes. d.p.i., days post infection; ELISA, enzyme-linked immunosorbent assay; p.o., perorally.

mice. However, it was still important to exclude the possibility that MNV-1 induces a very early antibody response that wanes at later times. To address this point, we compared the kinetics of the antiviral antibody response in $\mathrm{B} 6$ mice infected with either MNV-1 or MNV-3. Specifically, mice were infected with either $10^{4}$ (Figure 3a) or $10^{7}$ (Figure 3b) TCID $_{50}$ units MNV-1 or MNV-3. Fecal and serum samples were collected at $0,1,3,5,7,14,21$, and 28 d.p.i. and analyzed for virus-specific antibody. As expected, based on our previous results ${ }^{28}$, MNV-3 elicited significantly higher levels of fecal IgA, serum IgM, serum IgA, and serum IgG than MNV-1 under both dose conditions. The kinetics of the antibody responses generated to the two virus strains were similar: antiviral serum IgM was generally detectable by 3 d.p.i. and peaked at 7 d.p.i. although overall levels of this isotype remained modest throughout infection. Antiviral serum IgG was detectable at 5-7 d.p.i. and levels continued to increase through 28 d.p.i. Serum and fecal IgA were detectable at 7-14 d.p.i. and continued to increase through 28 d.p.i. Although there was detectable antiviral IgM and IgG in the serum of infected mice at 3-5 d.p.i., levels were expectedly very low and more importantly they were not higher in MNV-1-infected mice than in MNV-3-infected mice. Therefore, we conclude that these modest levels of virus-specific antibodies were unlikely to account for the increased MNV-1 titers observed in $\mu \mathrm{MT}$ mice.

\section{MNV-1, but not MNV-3, induces cytotoxic CD8 ${ }^{+} \mathrm{T}$ cells that function during acute infection}

Because we observed a role for B2M molecules in MNV-1 control (Figure 1d), we speculated that B cells act as APCs to stimulate $\mathrm{CD} 8{ }^{+} \mathrm{T}$ cells in response to MNV-1 infection. To test this, we first measured virus titers in $\mathrm{CD} 8^{-/-}$mice at 3 d.p.i. Precisely phenocopying results in $\mathrm{B}_{2} \mathrm{M}^{-1-}$ mice, $\mathrm{MNV}-1$ titers were higher in the distal ileum and MLNs, but not colon, of $\mathrm{CD} 8^{-1-}$ mice compared with $\mathrm{B} 6$ mice; whereas no difference was observed in any tissue of MNV-3-infected mice (Figure 4a). Because of its critical role as a cytotoxic effector molecule produced by $\mathrm{CD} 8{ }^{+} \mathrm{T}$ cells, we next measured the production of granzyme $\mathrm{B}(\mathrm{GrB})$ in various cell types in the Peyer's patches of infected mice. MNV-1 induced Peyer's patch $\mathrm{CD} 8{ }^{+} \mathrm{T}$ cells to express GrB at 3 d.p.i. whereas MNV-3 did not, as reflected by both an increased frequency of GrB-producing $\mathrm{CD}^{+} \mathrm{T}$ cells (Figure $\mathbf{4 b}$, left panel) and a 4.5 -fold increase in the total number of $\mathrm{GrB}^{+} \mathrm{CD}^{+} \mathrm{T}$ cells (Figure $4 \mathbf{b}$, right panel). No GrB induction was observed in Peyer's patch NK1.1 $1^{+}$natural killer cells (Figure $4 \mathrm{~b}$ ) or $\mathrm{CD} 4^{+} \mathrm{T}$ cells or $\mathrm{CD}_{19}{ }^{+} \mathrm{B}$ cells (data not shown) in response to either MNV-1 or MNV-3 infection. It was surprising to observe a critical role for $\mathrm{CD} 8{ }^{+} \mathrm{T}$ cells at such an early time post infection, raising the possibility that innate-like $\gamma \delta \mathrm{CD} 8^{+} \mathrm{T}$ cells that are abundant along the intestinal tract ${ }^{39}$ mediate early control of MNV-1. Although infection did not significantly alter the ratio of $\mathrm{CD}^{+}$ 
a

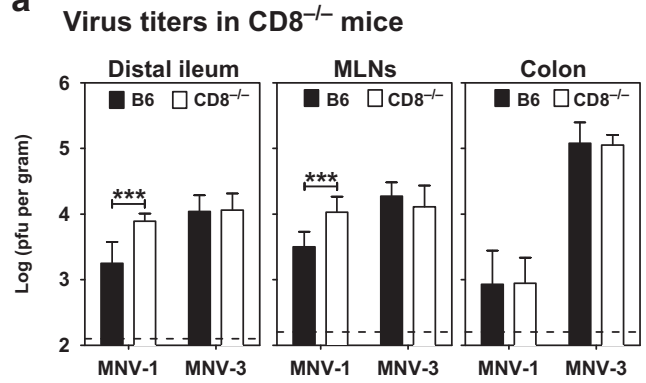

C

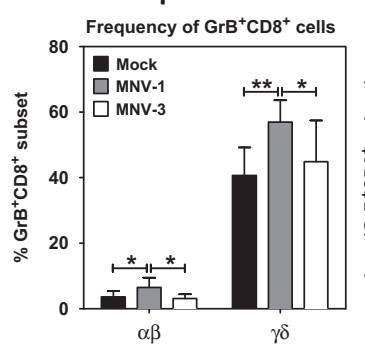

Total numbers of $\mathrm{GrB}^{+} \mathrm{CD} 8^{+}$cells

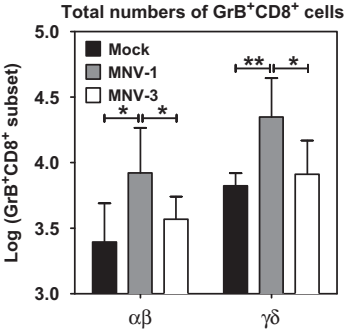

b

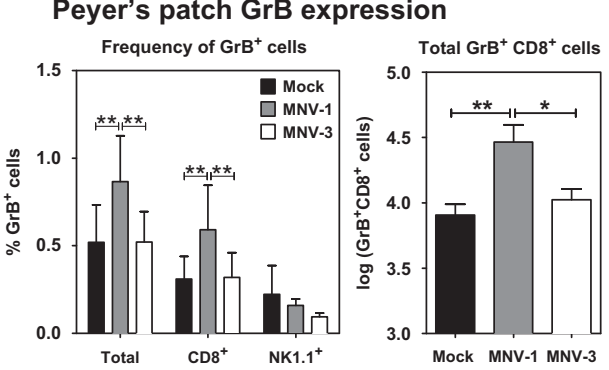

d

Figure 4 Cytotoxic CD8 ${ }^{+}$T cells control acute MNV-1, but not MNV-3, infection. (a) Groups of B6 (black bars) and CD8 ${ }^{-1-}$ (white bars) mice were infected p.o. with $10^{7} \mathrm{TCID}_{50}$ units MNV-1 or MNV-3. At 3 d.p.i., the indicated tissues were dissected and viral titers were determined by plaque assay. The data are reported as pfu per gram of tissue and the data for all mice per group ( $n=6-8$ mice over three experiments) are averaged. Dashed lines indicate the limit of detection for each tissue. The two mouse strains were compared for each tissue and virus strain for statistical purposes. (b) Peyer's patch cell suspensions prepared from groups ( $n=2-3$ ) of mock-inoculated B6 mice (black bars), or B6 mice infected p.o. for 3 days with $10^{7}$ TCID $_{50}$ units MNV-1 (gray bars) or MNV-3 (white bars), were stained for surface CD8, NK1.1, CD4, or CD19 and intracellular GrB. The experiment was repeated five times for a total of 13 replicates per condition. The data are presented as the percentage of the indicated cell populations expressing $\mathrm{GrB}$ in the left panel; no $\mathrm{GrB}$ was

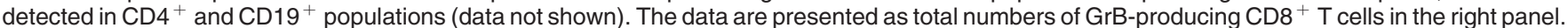
(c) Peyer's patch cell suspensions were stained for surface CD8 and the T cell receptor (TCR) $\beta$ chain (as an indication of $\alpha \beta$ T cells) or the TCR $\delta$ chain (as an indication of $\gamma \delta$ T cells), and intracellular GrB. The overall frequencies of $\alpha \beta$ and $\gamma \delta$ CD8 ${ }^{+}$T cells expressing GrB are presented in the left panel. The total numbers of the $\mathrm{GrB}$-expressing subsets are presented in the right panel. In panels $\mathbf{b}$ and $\mathbf{c}$, the mock group was compared with each virus group, and the two virus groups were compared, for statistical purposes. (d) IEL prepared from mock-inoculated (black bars) and MNV-1-infected (gray bars) wild-type B6 and B-cell-deficient $\mu \mathrm{MT}$ mice at 3 d.p.i. were stained for surface CD8 and intracellular $\mathrm{GrB}$. The experiment was repeated six times for a total of six replicates per condition. The data are presented as the total number of GrB-producing CD8 ${ }^{+}$IEL per condition. The mock group was compared with the infected group for each mouse strain for statistical purposes. d.p.i., days post infection; GrB, granzyme B; IEL, intestinal epithelial lymphocyte; p.o., perorally.

T-cell subsets in the Peyer's patches of infected mice (data not shown), the frequency of GrB-expressing $\alpha \beta$ and $\gamma \delta \mathrm{CD} 8^{+} \mathrm{T}$ cells was higher in MNV-1-infected, but not MNV-3-infected, mice (Figure 4c, left panel). Considering that there are far more $\alpha \beta \mathrm{CD}^{+}{ }^{+} \mathrm{T}$ cells in the Peyer's patches than $\gamma \delta \mathrm{CD} 8{ }^{+} \mathrm{T}$ cells, we also analyzed the total number of each subset expressing GrB. Although MNV-1 induced GrB expression in both subsets, there were quantitatively more GrB-expressing $\gamma \delta$ $\mathrm{CD} 8{ }^{+} \mathrm{T}$ cells than $\alpha \beta \mathrm{CD} 8^{+} \mathrm{T}$ cells in the Peyer's patches at 3 d.p.i. (Figure $4 c$, right panel).

To directly assess whether $\mathrm{B}$ cells are required for $\mathrm{CD} 8{ }^{+}$ T-cell activation during acute MNV-1 infection, we tested whether activation occurred in B-cell-deficient $\mu \mathrm{MT}$ mice. Because $\mu \mathrm{MT}$ mice do not develop Peyer's patches, we instead analyzed GrB levels in intestinal epithelial lymphocytes (IELs). Consistent with Peyer's patch results, there was a significant 3.2-fold increase in the number of GrB-producing $\mathrm{CD}^{+}$IEL in MNV-1-infected B6 mice (Figure 4d). In contrast, in spite of increased basal levels of GrB-producing $\mathrm{CD} 8^{+}$IEL in naive $\mu \mathrm{MT}$ mice compared with naive $\mathrm{B} 6$ mice, there was no appreciable increase induced by MNV-1 infection (Figure 4d), confirming that B cells are essential for activation. Consistent with a role for cytotoxic $\mathrm{CD} 8^{+} \mathrm{T}$ cells in $\mathrm{MNV}-1$ acute control, significantly higher virus titers were detected in the distal ileum, MLNs, and colon of GrA/B ${ }^{-1-}$ mice compared with their wild-type 129 counterparts at 3 d.p.i. (Figure 5a). Surprisingly though, perforin was dispensable for the early control of MNV-1 in all tissues (Figure 5b). Acute MNV-3 infection was not restricted by granzymes (Figure 5a) or perforin (Figure 5b). Altogether, these data illuminate a mechanism of innate immune control that requires $\mathrm{B} 2 \mathrm{M}, \mathrm{B}$ cells, granzymes, and $\mathrm{CD} 8^{+} \mathrm{T}$ cells, but not perforin, and that functions in a MuNoV strain-specific manner. Together with the observation that MNV-1 induced the expression of antigen-presenting molecules on $\mathrm{B}$ cells (Figure 1a-c), our data support a model in which MNV-1infected B cells present antigen on MHC class I molecules to stimulate cytotoxic $\mathrm{CD} 8{ }^{+} \mathrm{T}$ cells that control acute infection. We next investigated why this immune mechanism is not stimulated by MNV-3. 
a

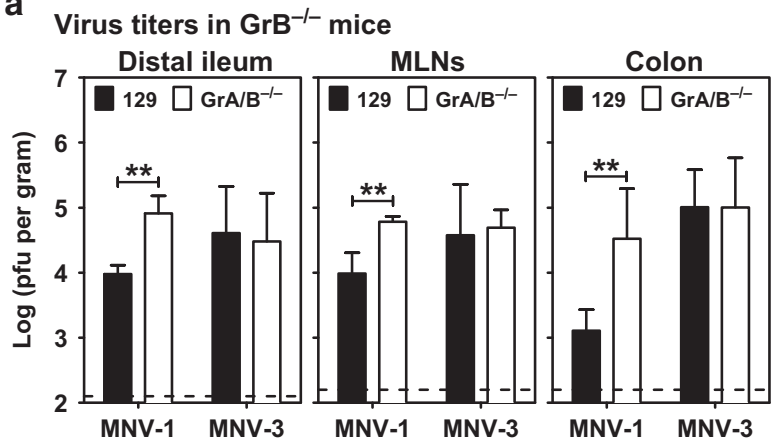

b

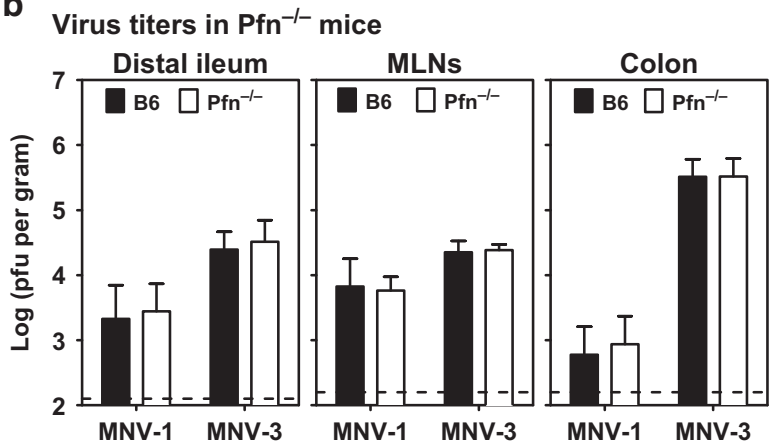

Figure 5 The control of acute MNV-1 infection requires granzymes but not perforin. (a) The same experiment described in Figure 4a was performed on groups of 129 (black bars) and $\mathrm{GrA} / \mathrm{B}^{-1-}$ (white bars) mice ( $n=6$ mice per group over two experiments). (b) The same experiment as described in Figure 4a was performed using B6 (black bars) and

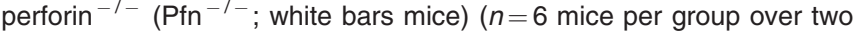
experiments). GrA/B, granzyme A/B.

\section{The minor structural protein VP2 regulates antigen} presentation molecule expression on B cells

We previously demonstrated that the viral minor structural protein VP2 regulates macrophage maturation in a MuNoVspecific manner, ${ }^{28}$ and so, we examined whether it similarly regulates B-cell maturation. When $\mathrm{M} 12$ cells were infected with MNV-1 expressing the MNV-3 VP2 protein (MNV-1.3VP2), there was significantly less MHC class I, MHC class II, CD40, CD80, and CD86 upregulation compared with cells infected with parental MNV-1 (Figure 6a, compare gray with white bars). Likewise, there was significantly more upregulation on cells infected with MNV-3 expressing the MNV-1 VP2 protein (MNV-3.1VP2) compared with parental MNV-3 (Figure 6a, compare vertically striped with diagonally striped bars). VP2 did not fully account for the regulation of antigen presentation molecules on cultured B cells, as was previously observed for macrophage maturation. ${ }^{28}$ Thus, VP2 partially regulates MuNoV-induced maturation of $\mathrm{B}$ cells and macrophages. Remarkably, our cumulative data here and in the study by Zhu et $a .^{28}$ demonstrate that VP2 acts not only in a virus strainspecific manner but also in a cell type-specific manner, with the MNV-1 VP2 protein blocking maturation in macrophages ${ }^{28}$ and the MNV-3 VP2 protein blocking maturation in B cells (Figure 6a). To test whether VP2 regulates B-cell-mediated control of acute MuNoV infection, B6 and $\mu \mathrm{MT}$ mice were infected with parental and VP2 chimeric viruses and titers determined at 3 d.p.i.. B-cell-mediated control was less effective in the distal ileum, MLNs, and colon of mice infected with MNV-1.3VP2 compared with parental MNV-1 (Figure 6b). Reciprocally, B cells controlled MNV-3.1VP2 infection in the distal ileum and MLNs in contrast to parental MNV-3, although no control was observed in the colon (Figure 6c). Overall, these data demonstrate that VP2 regulates the expression of antigen presentation molecules on B cells and B-cell-mediated control of acute infection.

\section{The intestinal microbiota does not contribute to the B-cell-mediated control of acute MNV-1 infection}

We and others have demonstrated that commensal bacteria enhance $\mathrm{MuNoV}$ infections in vivo, ${ }^{34,40,41}$ raising the possibility that the intestinal microbiota contributes to the virus strain-specific role of $\mathrm{B}$ cells in controlling acute $\mathrm{MuNoV}$ infection. To test this, we depleted the intestinal microbiota from B6 and $\mu \mathrm{MT}$ mice by administering a cocktail of four antibiotics (Abx) orally prior to viral infection. We have previously shown that Abx treatment of B6 mice results in a significant decrease in $\mathrm{MuNoV}$ titers at 1 d.p.i. ${ }^{34}$ Consistent with this, MNV-1 titers were reduced in Abx-treated B6 mice compared with phosphate-buffered saline-treated B6 controls at 3 d.p.i. (Figure 7). Titers were similarly reduced in Abxtreated $\mu \mathrm{MT}$ mice compared with untreated $\mu \mathrm{MT}$ controls. Importantly for our studies though, B cells contributed to the early control of MNV-1 even in the absence of commensal bacteria (Figure 7). Thus, we conclude that the B-cell-mediated early control of MNV-1 infection occurs independent of the intestinal microbiota.

\section{$B$ cells have distinct roles during early and late primary MNV-1 infections}

Because $\mu \mathrm{MT}$ mice failed to clear MNV-1 infection by 14 d.p.i., whereas B6 mice cleared infection by 7-10 d.p.i. (Figure 2a), we questioned whether other immune factors contributing to MNV-1 control at 3 d.p.i. would be similarly involved in viral clearance. Accordingly, we infected B6, $\mu \mathrm{MT}, \mathrm{CD}^{-1-}, \mathrm{B} 2 \mathrm{M}$, 129 , and $\mathrm{GrA} / \mathrm{B}^{-1-}$ mice with $\mathrm{MNV}-1$ and measured virus titers at 14 d.p.i. (Figure 8). Only B cells were required for MNV-1 clearance, distinguishing two independent functions for B cells in controlling and clearing acute MNV-1 infection. Neither of these functions was operational against MNV-3 infection in spite of the more robust antiviral antibody response elicited by this virus $\operatorname{strain}^{28}$ (and Figure 3 ).

\section{DISCUSSION}

Our collective data presented in this study are consistent with a model in which B cells act as APCs to activate $\mathrm{CD} 8{ }^{+}$T cells that function through a granzyme-dependent, perforin-independent mechanism to control MNV-1 infection at 3-5 d.p.i. This immune mechanism is not activated by the closely related MNV-3. When considering our observations in this study together with protective immunity findings in Zhu et al., ${ }^{28}$ our data illuminate an inverse correlation between control of acute 
a

VP2 regulates antigen presentation on B cells

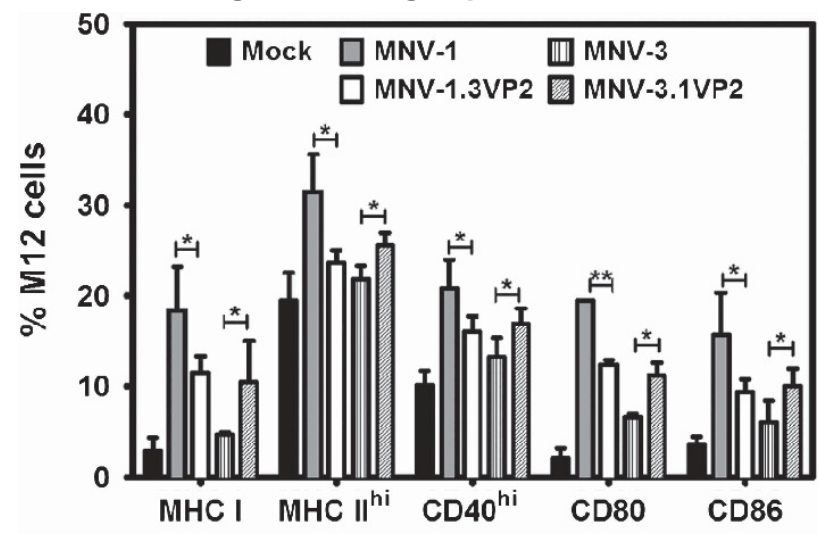

b

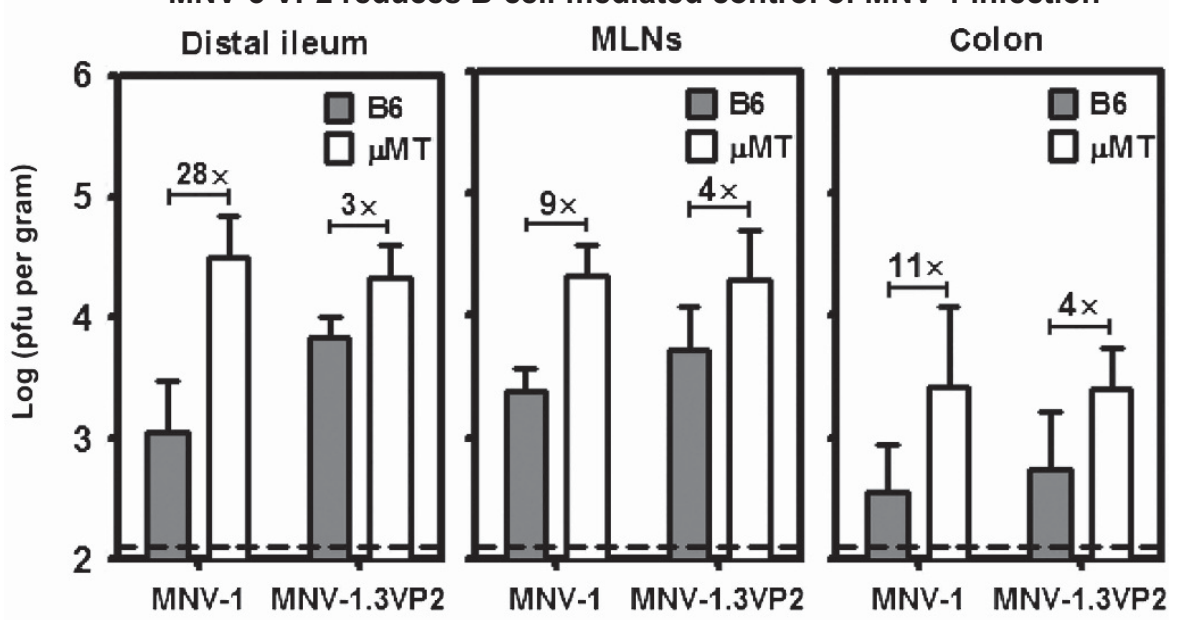

C

MNV-1 VP2 increases B-cell-mediated control of MNV-3 infection
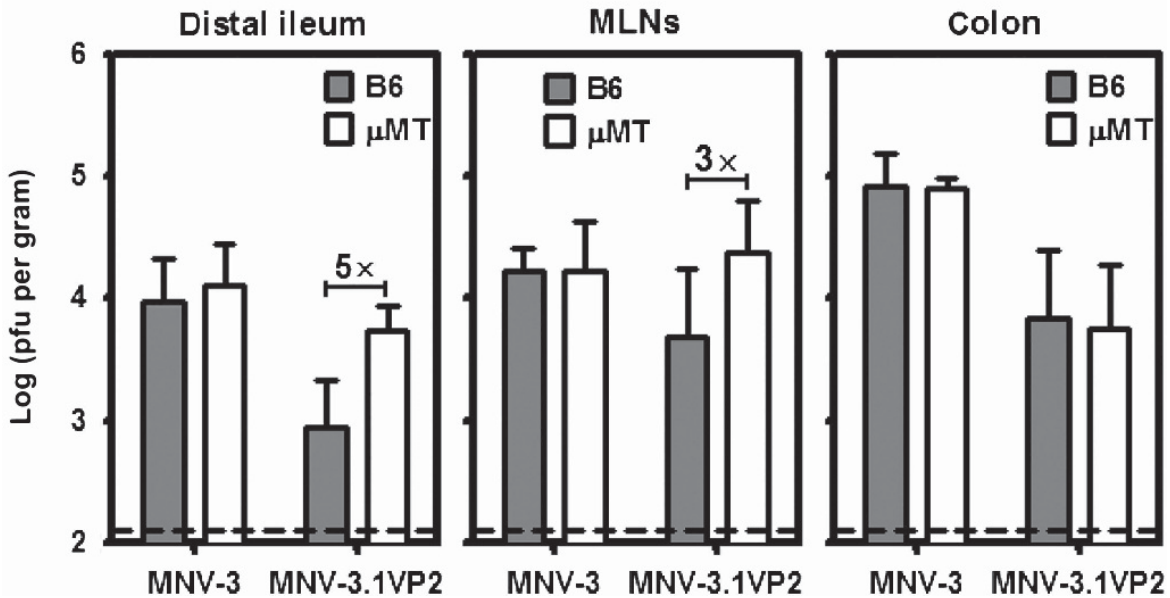

Figure 6 The VP2 protein regulates antigen presentation in B cells and B-cell-mediated control of acute MNV-1 infection. (a) Duplicate wells of M12 cells were inoculated with mock inoculum (black bars), MNV-1 (dark gray bars), MNV-1.3VP2 (white bars), MNV-3 (hatched bars, vertical lines), or MNV-3.1VP2 (hatched bars, diagonal lines) at MOI 5. At 2 d.p.i., cells were stained with antibodies to MHC I, MHC II, CD40, CD80, and CD86. Flow cytometry was carried out as described in the Methods and isotype control antibodies were used to set gates. The experiment was repeated three times. For all markers, the data from all experimental replicates are averaged. Statistical comparisons were made between each parental and chimeric pair. (b, c) Groups of B6 (gray bars) and $\mu \mathrm{MT}$ (white bars) mice were infected p.o. with $10^{7} \mathrm{TCID}_{50}$ units MNV-1 or MNV-1.3VP2 (b), or MNV-3 or MNV-3.1VP2 (c). At 3 d.p.i., the indicated tissues were dissected and viral titers were determined by plaque assay. The data are reported as pfu per gram of tissue and the data for all mice per group ( $n=5$ mice over two experiments) are averaged. Dashed lines indicate the limit of detection for each tissue. The fold-change in averaged virus titers comparing B6 to $\mu \mathrm{MT}$ for each virus strain is indicated above each set of bars. d.p.i., days post infection; MOI, multiplicity of infection; p.o., perorally. 


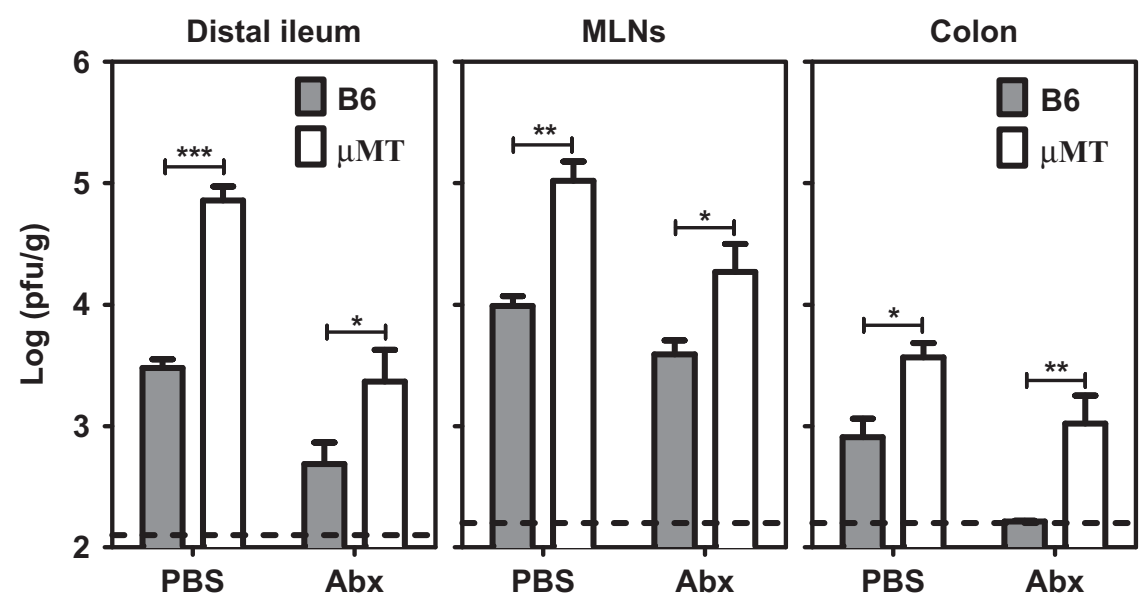

Figure 7 The B-cell-dependent control of acute MNV-1 infection occurs independent of the intestinal microbiota. Groups of B6 (gray bars) and $\mu \mathrm{MT}$ (white bars) mice were orally administered PBS or a cocktail of Abx, as described in the Methods and indicated on the $x$ axis. Both groups were then infected p.o. with $10^{7} \mathrm{TCID}_{50}$ units MNV-1. At 3 d.p.i., the indicated tissues were dissected and viral titers were determined by plaque assay. The data are reported as pfu per gram of tissue and the data for all mice per group $(n=8-11$ mice over four experiments) are averaged. Dashed lines indicate the limit of detection for each tissue. Statistical comparisons were made between B6 mice and $\mu \mathrm{MT}$ for each condition. Abx, antibiotics; d.p.i., days post infection; PBS, phosphate-buffered saline; p.o., perorally.

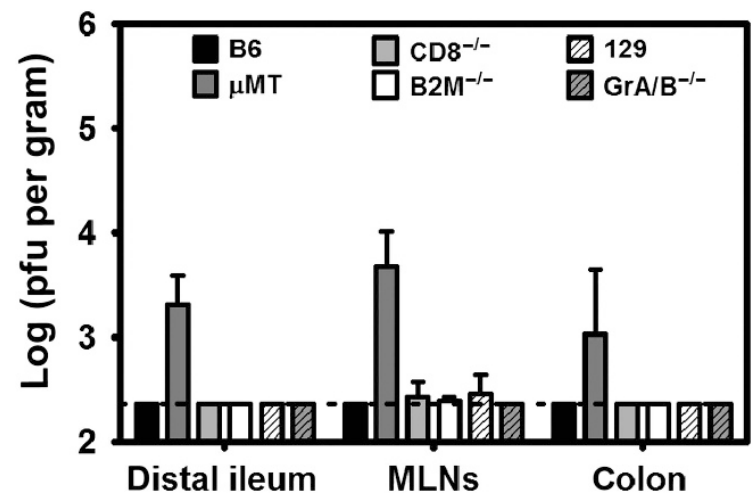

Figure 8 The role of $\mathrm{B}$ cells in MNV-1 clearance is independent of $\mathrm{CD} 8^{+}$ T cells, B2M, and GrA/B. Groups of B6 (black bars), $\mu \mathrm{MT}$ (dark gray bars), $\mathrm{CD}^{-1-}$ (light gray bars), B2M ${ }^{-1-}$ (white bars), 129 (white hatched bars), and $\mathrm{GrA} / \mathrm{B}^{-1-}$ (gray hatched bars) mice were infected p.o. with $10^{7}$ $\mathrm{TCID}_{50}$ units MNV-1. At 14 d.p.i., the tissues indicated on the $x$ axis were dissected and viral titers determined by plaque assay. The data are reported as pfu per gram of tissue and the data for all mice per group $(n=5$ per condition over two experiments) are averaged. d.p.i., days post infection; GrA/B, granzyme A/B; p.o., perorally.

MuNoV infection and protective immunity inductionMNV-1 induces an immune response(s) requiring $\mathrm{B}$ cells, $\mathrm{B} 2 \mathrm{M}, \mathrm{CD} 8{ }^{+} \mathrm{T}$ cells, and granzymes that controls acute infection but it fails to induce robust protective immunity; conversely, MNV-3 fails to activate the early immune response(s) required for controlling acute infection, but it does induce robust protective immunity dependent on antiviral antibody-secreting $\mathrm{B}$ cells and $\mathrm{CD} 4^{+} \mathrm{T}$ cells. Considering the high genetic similarity of MNV-1 and MNV-3, it is remarkable that they display such striking differences in immune outcome. It is important to recognize that $\mathrm{HuNoV}$ strains display much more substantial genetic diversity, and so, it is possible that they display an even broader range of interactions with the host immune system. The dichotomy of acute control vs. protective immunity induction appears to be dictated by the nature of the APC, with $\mathrm{B}$ cells presenting antigen on MHC class I stimulating early immune control and macrophages presenting antigen on MHC class II stimulating protective immunity. Moreover, we have revealed a central role for the MuNoV VP2 protein in regulating the cell type specificity of antigen presentation. Future studies will probe the mechanism by which VP2 regulates surface expression of antigen presentation molecules in a virus straindependent and cell type-specific manner. This information could directly inform next-generation vaccine development because ablation of VP2 antagonist activity could dramatically enhance immunogenicity of live attenuated viral vaccines.

One factor that could contribute to the differential immune responses elicited by MNV-1 and MNV-3 is the efficiency with which they replicate along the intestinal tract. Specifically, MNV-3 titers in wild-type mice were $\sim 1-\log$ higher in the distal ileum and MLNs, and $\sim 2$-logs higher in the colon, than MNV-1 titers at 3 d.p.i. (e.g., Figure 1d). However, several lines of evidence argue against this explanation: first, $\mathrm{MNV}-1$ and MNV-3 titers are actually much more comparable at the peak of infection (i.e., slightly higher MNV-1 titers in the distal ileum and $\sim 1-\log$ higher MNV-3 titers in the colon at 1 d.p.i. ${ }^{28}$ ), so the titers measured at 3 d.p.i. likely reflect differential immune control in contrast to a difference in the inherent ability of the virus strains to replicate in the intestines. Second, our in vivo phenotypes pertaining to antigen presentation regulation are perfectly mirrored during in vitro infections of cultured RAW 264.7 cells $^{28}$ and M12 cells (Figure 1a) where MNV-1 and MNV-3 replication kinetics are identical. ${ }^{34}$ This is true for VP2 chimeric viruses as well, where we previously demonstrated that parental and chimeric viruses replicate with identical kinetics in vitro yet there are significant differences in the upregulation of antigen presentation molecules regulated by 
VP2. ${ }^{28}$ Thus, differential in vivo replication efficiency is unlikely to account for the observed differences in immune response between MNV-1 and MNV-3.

The results reported in this study are generally consistent with previous findings in the field and extend our understanding of the immune response to primary MuNoV infections. For example, Chachu et al. ${ }^{42}$ previously reported that B cells control acute MNV-1 infection at 3 d.p.i. Our findings extend this observation by revealing virus strain specificity and by providing mechanistic insight into the role played by B cells at this very early time during infection. Specifically, our results support a role for B cells as APCs to stimulate cytotoxic $\mathrm{CD} 8{ }^{+} \mathrm{T}$ cells that control acute infection. Second, Tomov et al. ${ }^{43}$ demonstrated that antiviral CD8 ${ }^{+} \mathrm{T}$ cells are detectable by 8 d.p.i. in MNV-1-infected mice and that this response is quantitatively and qualitatively superior to the $\mathrm{CD}^{+}{ }^{+} \mathrm{T}$-cell response to another MuNoV strain called CR6. Our results indicate a role for $\mathrm{CD}^{+}{ }^{+} \mathrm{T}$ cells in MNV-1 control as early as 3 d.p.i., and so, it will be critical in future studies to determine whether these $T$ cells are virus-specific or instead are innate-like $\mathrm{CD} 8^{+} \mathrm{T}$ cells such as $\gamma \delta \mathrm{T}$ cells. Moreover, they suggest that suboptimal $\mathrm{CD}^{+}{ }^{+}$T-cell activation by certain MuNoV strains is related to antagonism of B-cell maturation as APCs.

Several additional important conclusions can be drawn from the results presented in this study. First, although B cells (Figure 2a) and granzymes (Figure 5a) were critical for MNV1 control in the small and large intestines, B2M (Figure 1d) and $\mathrm{CD}^{+} \mathrm{T}$ cells (Figure 4a) were important for control in the distal ileum and MLNs that drain the small intestine but dispensable in the colon. These data illuminate a distinction in the immune mechanisms controlling MNV-1 infection in different regions of the intestine, adding to a growing body of evidence for intestinal immune compartmentalization. ${ }^{44}$ The MuNoV model system provides a unique opportunity to probe regional distinctions along the intestinal tract considering that $\mathrm{MuNoV}$ strains display variable preference for specific regions of the intestine (e.g., MNV-1 reaches higher peak titers in the small intestine, whereas MNV-3 reaches higher peak titers in the large intestine ${ }^{28}$ ); and our and others ${ }^{45}$ results show that they are controlled by regionally variable immune responses. Second, our results provide general insight into the role of $\mathrm{B}$ cells as APCs in stimulating $\mathrm{CD} 8^{+}{ }^{-} \mathrm{T}$-cell responses: $\mathrm{B}$ cells are well-established APCs but they predominantly present peptides in complex with MHC class II molecules to $\mathrm{CD} 4{ }^{+} \mathrm{T}$ cells following internalization of antigens bound by the B-cell receptor. ${ }^{36}$ There is increasing interest in understanding the capacity of $\mathrm{B}$ cells to prime $\mathrm{CD} 8^{+} \mathrm{T}$-cell responses because of their potential use in cell-based immunotherapy (e.g., refs 46,47). We revealed that B cells can rapidly activate $\mathrm{CD}^{+} \mathrm{T}$ cells in the apparent absence of $\mathrm{CD} 4{ }^{+} \mathrm{T}$-cell activation when they are infected with a cytosolic RNA virus. Third, we demonstrated that granzymes contribute to MNV-1 early control (Figure 5a) while perforin is dispensable (Figure 5b). Although granzymes are most well-established to function in concert with perforin to induce apoptosis of target cells, they are increasingly recognized to have perforin-independent functions that include direct cleavage of viral proteins, cleavage of cell surface receptors and extracellular matrix components, and induction of proinflammatory cytokines. ${ }^{4-51}$ Future studies will probe the precise mechanism of granzyme-mediated control of MNV-1 infection along the intestinal tract. Finally, we revealed that $\mathrm{B}$ cells have a distinct role in MNV-1 clearance at 7-14 d.p.i. that does not require $\mathrm{B} 2 \mathrm{M}, \mathrm{CD} 8$, or granzymes (Figure 8). Although it is tempting to speculate that this role for $\mathrm{B}$ cells involves their production of antiviral antibody, MNV-3 induces a stronger antibody response yet it is not cleared acutely but instead establishes persistence. ${ }^{28,37,38}$

In conclusion, our findings demonstrate that two highly genetically related intra-cluster MuNoV strains possess distinct strategies to antagonize host immune responses. Although both viruses prevent upregulation of antigen presentation molecules on infected cells, they do so in a cell type-specific manner. While prevention of macrophage antigen presentation results in impaired protective immunity induction, ${ }^{28}$ prevention of B-cell antigen presentation results in impaired control of acute infection. Remarkably, the viral VP2 protein responsible for this antagonist activity thus functions in a virus straindependent and cell type-specific manner. There are 18 amino acid differences between MNV-1 and MNV-3 VP2 proteins that could account for functional distinctions (Supplementary Figure S1 online); future studies will probe the contribution of individual residues in regulating antigen presentation in macrophages and B cells. Elucidating key viral and host mechanisms regulating the overall outcome of norovirus infections may inform future vaccine design.

\section{METHODS}

Cells and viruses. The M12, RAW 264.7, and 293T cell lines were maintained in Dulbecco's modified Eagle medium (Lonza, Basel, Switzerland) supplemented with $10 \%$ fetal calf serum (Sigma, St. Louis, MO, Cat. \#F6178), $100 \mathrm{U}$ penicillin/ml, and $100 \mu \mathrm{g} \mathrm{ml} \mathrm{streptomycin.}$ Stocks of recombinant MNV-1.CW3 (GenBank accession number KC782764) and MNV-3 (GenBank accession number KC792553), and the chimeric MNV-1.3VP2 and MNV-3.1VP2 viruses in which the ORF3 gene encoding VP2 was swapped between the two virus strains, were generated as described previously. ${ }^{28}$ Briefly, $10^{6} 293 \mathrm{~T}$ cells were transfected with $5 \mu \mathrm{g}$ infectious clone using Lipofectamine 2000 (Life Technologies, Carlsbad, CA), cells were freeze-thawed after 1 day, lysates titrated with a standard TCID $_{50}$ assay, ${ }^{52}$ and RAW 264.7 cells infected at multiplicity of infection (MOI) 0.05. RAW 264.7 lysates were freeze-thawed when cultures displayed $90 \%$ cytopathic effect and supernatants were clarified by low-speed centrifugation followed by purification through a $25 \%$ sucrose cushion. A mock inoculum stock was prepared in parallel using RAW 264.7 lysate from uninfected cultures. The viral genomes of stocks were sequenced completely to confirm no mutations arose during stock generation.

M12 cell infection and flow cytometry. M12 cells inoculated with mock inoculum; recombinant MNV-1, MNV-1.3VP2, MNV-3, or MNV-3.1VP2 at MOI 5; or UV-inactivated MNV-1 or MNV-3 were incubated at $37^{\circ} \mathrm{C}$ for 2 days and then stained with antibodies directed against MHC class I, MHC class II, CD40, CD80, and CD86 (eBioscience, San Diego, CA). RAW 264.7 cells were infected for 1 day prior to flow cytometry on the basis of their more rapid replication kinetics. ${ }^{34}$ Matched isotype controls were used for all antibodies to set gates. Flow cytometric analysis was performed on a FACSCalibur 
instrument (BD Biosciences, San Jose, CA) and data were analyzed using FCS Express 4 software.

Mice and infections. Specific-pathogen-free mice used in this study were bred and housed in animal facilities at the University of Florida. Wild-type B6 mice (Jackson, Bar Harbor, ME, \#000664) and the following knockout strains on the B6 background were used in these studies: B-cell-deficient ( $\mu \mathrm{MT}$, Jackson \#002288), perforin-deficient (Pfn $^{-1-}$, Jackson \#002407), CD8-deficient $\left(\mathrm{CD} 8^{-1-}\right.$, Jackson \#002665), B2M-deficient (B2M ${ }^{-1-}$, Jackson \#002087), and MHC class II-deficient (MHC II ${ }^{-/-}$, Jackson \#003584) strains. Wild-type 129X1/SvJ mice (129, Jackson \#000691) and granzyme A/B-deficient mice on a 129 background $\left(\mathrm{GrA} / \mathrm{B}^{-1-}\right.$, Jackson \#010608) were also used. For all acute infection experiments, 6-8-week old, sex-matched mice were inoculated perorally (p.o.) with $10^{7} \mathrm{TCID}_{50}$ units of the indicated virus strain in $25 \mu \mathrm{l}$ inoculum, unless otherwise indicated. For virus load determination, tissue samples were harvested at the indicated time points and titrated by plaque assay, as previously described. ${ }^{27,28}$ The intestinal microbiota was depleted from mice as described previously. ${ }^{34}$ Briefly, mice were administered daily a cocktail of $10 \mathrm{mg}$ each of ampicillin (Acros Organics, Waltham, MA), neomycin (Sigma), metronidazole (Acros Organics), and vancomycin (Fisher Scientific, Waltham, MA) via oral gavage for 5 days. After day 5 , antibiotics $(\mathrm{Abx})$ were added to the drinking water at a concentration of $1 \mathrm{gl}^{-1}$ for ampicillin, metronidazole, and neomycin and $500 \mathrm{mgl}^{-1}$ for vancomycin. To confirm efficient depletions, fecal samples were collected from mice at the fifth day of gavage, homogenized, plated on brain-heart infusion agar with $10 \%$ sheep blood, and cultured under anaerobic conditions at $37^{\circ} \mathrm{C}$ for 2 days followed by aerobic conditions at $37^{\circ} \mathrm{C}$ for 1 day. Abx were maintained in the drinking water throughout the entire experiment and the infections were only carried out after the Abx-treated animals were verified to be free of detectable intestinal bacteria.

Peyer's patch and intestinal epithelial lymphocyte (IEL) isolations and flow cytometry. Peyer's patches were dissected from small intestines and single cell suspensions were generated by grinding the tissues through a $70-\mu \mathrm{m}$ cell strainer in $3 \mathrm{ml}$ RPMI medium supplemented with $10 \%$ fetal bovine serum. IELs were isolated using a protocol adapted from Graves et al. ${ }^{53}$ After removal of fat and Peyer's patches from the small intestine, the tissue was opened longitudinally, washed with cold phosphate-buffered saline, cut into 1-2-cm-long pieces, and placed into $35 \mathrm{ml}$ ice-cold $\mathrm{Mg}^{2+}$ - and $\mathrm{Ca}^{2+}$-free Hank's Balanced Salt Solution wash buffer supplemented with $100 \mathrm{U}$ penicillin, $100 \mu \mathrm{g} \mathrm{ml}^{-1}$ streptomycin, and $0.5 \mathrm{mM}$ dithiothreitol. The tissue was then inverted vigorously 10 times and allowed to settle for $1 \mathrm{~min}$. The supernatant was collected and the intestinal tissue was washed an additional four times. The combined supernatant was centrifuged at $100 \mathrm{~g}$ for $2 \mathrm{~min}$ to remove large tissue debris, passed over a $100-\mu \mathrm{m}$ cell strainer, centrifuged at $400 \mathrm{~g}$ for $10 \mathrm{~min}$, and the pellet resuspended in complete RPMI. The resulting IEL suspension was filtered through a $70-\mu \mathrm{m}$ cell strainer and subjected to flow cytometric analysis. For flow cytometry, cells were resuspended in $1 \times$ phosphatebuffered saline containing 5\% sodium azide, 5\% bovine serum albumin, and Fc Block antibody (anti-mouse CD16/32 purified, Cat. \#14-0161-86, eBioscience) to a final concentration of $2 \times 10^{7}$ cells per milliliter. After a 30-min incubation, cells were stained for surface markers using antibodies to CD19, MHC class I, MHC class II, CD40, CD80, CD86, CD4, CD8, NK1.1, TCR $\beta$ chain, or TCR $\delta$ chain (BD Biosciences). Cells were fixed and permeabilized using the Cytofix/Cytoperm kit (BD Biosciences) and stained for intracellular GrB. Matched isotype controls were used for all antibodies and used to set gates. Flow cytometry and data analysis was performed as mentioned above.

Virus-specific enzyme-linked immunosorbent assay. Serum samples were collected by submandibular puncture and fecal pellets $(0.05 \mathrm{~g})$ were collected into $0.5 \mathrm{ml}$ phosphate-buffered saline containing protease inhibitor cocktail (Sigma), homogenized, and centrifuged to generate a fecal lysate. Serum samples and fecal lysates were subjected to a virus strain-specific enzyme-linked immunosorbent assay that has been previously described. ${ }^{28,54}$ In brief, 96-well plates were coated with $250 \mathrm{ng}$ MNV-1 or MNV-3 recombinant VP1/VP2 (rVP1/2) protein and incubated overnight at $4{ }^{\circ} \mathrm{C}$. Serum samples diluted 1:20 and fecal lysates diluted 1:10 were applied, followed by anti-mouse IgA for fecal lysates or anti-mouse IgM, IgG, or IgA for serum samples. Each secondary antibody was conjugated to horseradish peroxidase. The ABTS $\left(2,2^{\prime}\right.$-azinobis(3-ethylbenzthiazolinesulfonic acid)) substrate was added and absorbance values read at $415 \mathrm{~nm}$ using a Spectramax M2 plate reader (Molecular Devices, Sunnyvale, USA). A standard curve was generated for each plate using serial dilutions of a positive control MNV-1 or MNV-3 serum sample to ensure plate-to-plate consistency.

Statistical analyses. All data were analyzed with GraphPad Prism software. Error bars denote standard deviation in all figures and $P$ values were determined using two-tailed Student's $t$-tests for flow cytometry data and Mann-Whitney tests for tissue titer comparisons $\left({ }^{\star} P<0.05,{ }^{* \star} P<0.01,{ }^{* * *} P<0.001\right)$.

SUPPLEMENTARY MATERIAL is linked to the online version of the paper at http://www.nature.com/mi

\section{ACKNOWLEDGMENTS}

This work was funded by NIH R01Al116892 and R01Al081921.

\section{DISCLOSURE}

The authors declared no conflict of interest.

(c) 2016 Society for Mucosal Immunology

\section{REFERENCES}

1. Koo, H.L. et al. Noroviruses: the most common pediatric viral enteric pathogen at a large university hospital after introduction of rotavirus vaccination. J. Pediatr. Infect. Dis. Soc 2, 57-60 (2013).

2. Payne, D.C. et al. Norovirus and medically attended gastroenteritis in U.S. children. N. Engl. J. Med. 368, 1121-1130 (2013).

3. Patel, M.M. Systematic literature review of role of noroviruses in sporadic gastroenteritis. Emerg. Infect. Dis. 14, 1224-1231 (2008).

4. Ahmed, S.M. et al. Global prevalence of norovirus in cases of gastroenteritis: a systematic review and meta-analysis. Lancet Infect. Dis. 14, 725-730 (2014).

5. CDC - 2011 Estimates of Foodborne Illness [Internet]. 2011. Available from http://www.cdc.gov/foodborneburden/2011-foodborne-estimates.html.

6. Scharff, R.L. Health-related costs from foodborne illness in the United States. Produce Safety Project Georgetown University, 2010.

7. Parrino, T.A., Schreiber, D.S., Trier, J.S., Kapikian, A.Z. \& Blacklow, N.R. Clinical immunity in acute gastroenteritis caused by Norwalk agent. N. Engl. J. Med. 297, 86-89 (1977).

8. Johnson, P.C., Mathewson, J.J., DuPont, H.L. \& Greenberg, H.B. Multiplechallenge study of host susceptibility to Norwalk gastroenteritis in US adults. J. Infect. Dis. 161, 18-21 (1990).

9. Green, K.Y. Caliciviridae: the noroviruses. In: Fields Virology, 6th edn. Lippincott, Williams, and Wilkins: Philadelphia, PA, USA, 2013. pp 582-608.

10. Atmar, R.L. et al. Norovirus vaccine against experimental human norwalk virus illness. N. Engl. J. Med. 365, 2178-2187 (2011).

11. Bernstein, D.I. et al. Norovirus vaccine against experimental human Gll.4 virus illness: a challenge study in healthy adults. J. Infect. Dis. 211, 870-878 (2015).

12. Lindesmith, L.C. et al. Broad blockade antibody responses in human volunteers after immunization with a multivalent norovirus vip candidate vaccine: immunological analyses from a phase I clinical trial. PLoS Med. 12, e1001807 (2015).

13. Roth, A.N. \& Karst, S.M. Norovirus mechanisms of immune antagonism. Curr. Opin. Virol. 16, 24-30 (2016).

14. Sharp, T.M., Guix, S., Katayama, K., Crawford, S.E. \& Estes, M.K. Inhibition of cellular protein secretion by norwalk virus nonstructural protein p22 
requires a mimic of an endoplasmic reticulum export signal. PloS One 5, e13130 (2010).

15. Ettayebi, K. \& Hardy, M.E. Norwalk virus nonstructural protein $\mathrm{p} 48$ forms a complex with the SNARE regulator VAP-A and prevents cell surface expression of vesicular stomatitis virus G protein. J. Virol. 77, 11790-11797 (2003).

16. Taube, S. et al. A mouse model for human norovirus. mBio 4, e00450-13 (2013).

17. Cheetham, S., Souza, M., Meulia, T., Grimes, S., Han, M.G. \& Saif, L.J. Pathogenesis of a genogroup II human norovirus in gnotobiotic pigs. J. Virol. 80, 10372-10381 (2006).

18. Souza, M., Azevedo, M.S., Jung, K., Cheetham, S. \& Saif, L.J. Pathogenesis and immune responses in gnotobiotic calves after infection with the genogroup II.4-HS66 strain of human norovirus. J. Virol. 82, 1777-1786 (2008).

19. Bok, K. et al. Chimpanzees as an animal model for human norovirus infection and vaccine development. Proc. Natl Acad. Sci. USA 108, 325-330 (2011).

20. Karst, S.M., Wobus, C.E., Lay, M., Davidson, J. \& Virgin, H.W. STAT1-dependent innate immunity to a norwalk-like virus. Science 299, 1575-1578 (2003).

21. Karst, S.M., Wobus, C.E., Goodfellow, I.G., Green, K.Y. \& Virgin, H.W. Advances in norovirus biology. Cell Host Microbe. 15, 668-680 (2014).

22. Blacklow, N.R. et al. Acute infectious nonbacterial gastroenteritis: etiology and pathogenesis. Ann. Intern. Med. 76, 993-1008 (1972).

23. Dolin, R., Levy, A.G., Wyatt, R.G., Thornhill, T.S. \& Gardner, J.D. Viral gastroenteritis induced by the Hawaii agent. Jejunal histopathology and serologic response. Am. J. Med. 59, 761-768 (1975).

24. Schreiber, D.S., Blacklow, N.R. \& Trier, J.S. The mucosal lesion of the proximal small intestine in acute infectious nonbacterial gastroenteritis. N. Engl. J. Med. 288, 1318-1323 (1973).

25. Mumphrey, S.M. et al. Murine norovirus 1 infection is associated with histopathological changes in immunocompetent hosts, but clinical disease is prevented by STAT1-dependent interferon responses. J. Virol. 81, 3251-3263 (2007).

26. Troeger, $\mathrm{H}$. et al. Structural and functional changes of the duodenum in human norovirus infection. Gut 58, 1070-1077 (2009).

27. Kahan, S.M., Liu, G., Reinhard, M.K., Hsu, C.C., Livingston, R.S. \& Karst, S.M. Comparative murine norovirus studies reveal a lack of correlation between intestinal virus titers and enteric pathology. Virology 421, 202-210 (2011).

28. Zhu, S. et al. Identification of immune and viral correlates of norovirus protective immunity through comparative study of intra-cluster norovirus strains. PLoS Pathog. 9, e1003592 (2013).

29. Liu, G., Kahan, S.M., Jia, Y. \& Karst, S.M. Primary high-dose murine norovirus 1 infection fails to protect from secondary challenge with homologous virus. J. Virol. 83, 6963-6968 (2009).

30. McFadden, N. et al. Norovirus regulation of the innate immune response and apoptosis occurs via the product of the alternative open reading frame 4. PLoS Pathog. 7, e1002413 (2011).

31. Wobus, C.E. et al. Replication of norovirus in cell culture reveals a tropism for dendritic cells and macrophages. PLoS Biol. 2, e432 (2004).

32. Lay, M.K. et al. Norwalk virus does not replicate in human macrophages or dendritic cells derived from the peripheral blood of susceptible humans. Virology 406, 1-11 (2010).

33. Chan, M.C.-W., Ho, W.-S. \& Sung, J.J.-Y. In vitro whole-virus binding of a norovirus genogroup II genotype 4 strain to cells of the lamina propria and brunner's glands in the human duodenum. J. Virol. 85, 8427-8430 (2011).
34. Jones, M.K. et al. Enteric bacteria promote human and murine norovirus infection of B cells. Science 346, 755-759 (2014).

35. Karst, S.M. Identification of a novel cellular target and a co-factor for norovirus infection - B cells \& commensal bacteria. Gut Microbes 6 , 266-271 (2015).

36. Rodríguez-Pinto, D. B cells as antigen presenting cells. Cell Immunol. 238, 67-75 (2005).

37. Hsu, C.C., Riley, L.K., Wills, H.M. \& Livingston, R.S. Persistent infection with and serologic crossreactivity of three novel murine noroviruses. Comp. Med 56, 247-251 (2006).

38. Arias, A., Bailey, D., Chaudhry, Y. \& Goodfellow, I.G. Development of a reverse genetics system for murine norovirus 3; long-term persistence occurs in the caecum and colon. J. Gen. Virol. 93 (Pt 7), 1432-1441 (2012).

39. Vantourout, P. \& Hayday, A. Six-of-the-best: unique contributions of $\gamma \delta$ T cells to immunology. Nat. Rev. Immunol. 13, 88-100 (2013).

40. Baldridge, M.T. et al. Commensal microbes and interferon- $\lambda$ determine persistence of enteric murine norovirus infection. Science 347, 266-269 (2015).

41. Kernbauer, E., Ding, Y. \& Cadwell, K. An enteric virus can replace the beneficial function of commensal bacteria. Nature 516, 94-98 (2014).

42. Chachu, K.A., Strong, D.W., LoBue, A.D., Wobus, C.E., Baric, R.S. \& Virgin, H.W. Antibody is critical for the clearance of murine norovirus infection. J. Virol. 82, 6610-6617 (2008).

43. Tomov, V.T. et al. Persistent enteric murine norovirus infection is associated with functionally suboptimal virus-specific CD8 T cell responses. J. Virol. 87, 7015-7031 (2013).

44. Mowat, A.M. \& Agace, W.W. Regional specialization within the intestinal immune system. Nat. Rev. Immunol. 14, 667-685 (2014).

45. Chachu, K.A., LoBue, A.D., Strong, D.W., Baric, R.S. \& Virgin, H.W. Immune mechanisms responsible for vaccination against and clearance of mucosal and lymphatic norovirus infection. PLoS Pathog. 4, e1000236 (2008).

46. Lee Szeto, G. et al. Microfluidic squeezing for intracellular antigen loading in polyclonal B-cells as cellular vaccines. Sci. Rep. 5, 10276 (2015).

47. Ahmadi, T., Flies, A., Efebera, Y. \& Sherr, D.H. CD40 ligand-activated, antigen-specific B cells are comparable to mature dendritic cells in presenting protein antigens and major histocompatibility complex class Iand class II-binding peptides. Immunology 124, 129-140 (2008).

48. Hagn, M. \& Jahrsdorfer, B. Why do human B cells secrete granzyme B? Insights into a novel B-cell differentiation pathway. Oncoimmunology $\mathbf{1}$, 1368-1375 (2012).

49. Spencer, C.T. et al. Granzyme A produced by $\gamma 9 \delta 2$ T cells induces human macrophages to inhibit growth of an intracellular pathogen. PLoS Pathog. 9, e1003119 (2013).

50. Andrade, F. Non-cytotoxic antiviral activities of granzymes in the context of the immune antiviral state. Immunol. Rev. 235, 128-146 (2010).

51. Romero, V. \& Andrade, F. Non-apoptotic functions of granzymes. Tissue Antigens 71, 409-416 (2008).

52. Thackray, L.B. et al. Murine noroviruses comprising a single genogroup exhibit biological diversity despite limited sequence divergence. J. Virol. 81 , 10460-10473 (2007).

53. Graves, C.L. et al. A method for high purity intestinal epithelial cell culture from adult human and murine tissues for the investigation of innate immune function. J. Immunol. Methods 414, 20-31 (2014).

54. Hickman, $\mathrm{D}$ et al. The effect of malnutrition on norovirus infection. mBio 5, e01032-13 (2014). 